\title{
A Probabilistic Energy-Aware Model for Mobile Ad-Hoc Networks
}

\author{
Lucia Gallina, Sardaouna Hamadou, Andrea Marin, and Sabina Rossi \\ Università Ca' Foscari, Venezia (Italy) \\ \{lgallina, sh, marin, srossi\}@dais . unive.it
}

\begin{abstract}
We propose a probabilistic, energy-aware, broadcast calculus for the analysis of mobile ad-hoc networks. The semantics of our model is expressed in terms of Segala's probabilistic automata driven by schedulers to resolve the nondeterministic choice among the probability distributions over target states. We develop a probabilistic observational congruence and a energy-aware preorder semantics. The observational congruence allows us to verify whether two networks exhibit the same observable probabilistic behaviour (connectivity), while the preorder is used to compare the energy consumption of different, but behaviourally equivalent, networks. As an application, we analyse and compare the energy consumption of two well-known automatic repeat request (ARQ)based error control protocols: stop-and-wait (SW) and go-back-N (GBN).
\end{abstract}

\section{Introduction}

Mobile ad-hoc networks (MANETs) consist of a collection of nodes that communicate with each other through wireless links without a pre-established networking infrastructure. A common feature of most of these networks is free node mobility: each device in a MANET is free to move independently in any direction, and will therefore change its links to other devices frequently. These changes in the network topology can cause the nodes to continuously enter and exit each other transmission area and hence highly dynamic routing algorithms are needed to ensure the connectivity. Moreover, mobile devices may have strict requirements on the energy consumption because their expected life-time often depends on the energy stored in a battery or other exhaustible power sources. Hence, the communication protocols must face the problem of providing a full connectivity among the network devices while maintaining good performance both in terms of throughput and of energy conservation (see, e.g., $[10,12]$ ).

The definition of a general formalism capable of expressing both qualitative (connectivity) and quantitative (power consumption and throughput) analysis is a challenging topic of research.

In this paper we define a calculus, named Probabilistic E-BUM, for formally reasoning about probabilistic energy-aware broadcast, unicast and multicast communications of mobile ad-hoc networks. This is an extension of the E-BUM calculus [3] where probability distributions are used to describe the movements of nodes. Our calculus allows us to model the ability of a node to 
broadcast a message to any other node within its physical transmission range, and to move in and out of the transmission range of other nodes in the network. The connectivity of a node is represented by a location and a transmission radius. Broadcast communications are limited to the transmission cell of the sender, while unicast and multicast communications are modelled by specifying, for each output action, the addresses of the intended recipients of the message. Moreover, the possibility for a node to control its transmission power is modeled by enabling nodes to modify the transmission radius of their communications.

Probabilistic E-BUM calculus deals with both nondeterministic and probabilistic choices. Its semantics is expressed in terms of Segala's probabilistic automata [9] driven by schedulers to resolve the nondeterministic choice among the probability distributions over target states. In this paper we propose a notion of probabilistic observational congruence in the style of [7] and also define a labelled semantics to model the interactions of the system with the surrounding environmnet. We provide a labelled bisimilarity as an efficient proof method for the observational congruence. Intuitively, two networks are deemed equivalent if they exhibit the same probabilistic behaviour (connectivity) relative to the corresponding set of intended recipients and to a specific set of schedulers. Moreover, based on the labelled semantics we define an energy-aware preorder over networks which allows us to compare the average energy cost of different networks but exhibiting the same connectivity behaviour. This property can be used to replace a network component with a less energy consuming one while maintaining connectivity. As an application we analyse and compare the energy consumption of two well-known automatic repeat request (ARQ)-based error control protocols: stop-and-wait (SW) and go-back-N (GBN).

Related works. Probabilistic and stochastic models are nowadays widely used in the design and verification of complex systems. Song and Godskesen [11] proposed a probabilistic broadcast calculus for mobile and wireless networks whose connections are unreliable. The peculiarity of this calculus is the introduction of a probabilistic mobility function which defines the mobility rules of the connections. Palamidessi et al. [?] defined an extension of the applied pi-calculus with nondeterministic and probabilistic choice operators, while Priami [6] introduced a stochastic extension of the pi-caluclus which allows one to describe dynamically reconfigurable or mobile networks. Another important stochastic process algebra for performance evalutation is PEPA [4], introduced by Jane Hillston at al. which is used for modelling systems composed of concurrently active components which co-operate and share work. Here cooperation is modelled in a CSP style, using share names. Bernardo et al. introduced EMPA ${ }_{g r}$ [1], an extended Markovian process algebra including probabilities, priority and exponentially distributed durations. The peculiarity of this calculus is the modelling of exponentially timed as well as immediate actions, whose selection is control by a priority level associated with them.

As far as energy consumption is concerned, several papers address the problem of studying the energy consumption of a specific communication protocol for wireless networks. For instance, in [12] the authors define a Markov Reward 


\begin{tabular}{llcl}
\hline Networks & & Processes & \\
$\mathrm{M}, \mathrm{N}::=\mathbf{0}$ & Empty network & $\mathrm{P}, \mathrm{Q}, \mathrm{R}::=\mathbf{0}$ & Inactive process \\
$\left|M_{1}\right| M_{2}$ & Parallel composition & $\mid c(\tilde{x}) . P$ & Input \\
$\mid n[P]_{l}$ & Node (or device) & $\mid \bar{c}_{L, r}\langle\tilde{w}\rangle . P$ & Output \\
& & $\mid\left[w_{1}=w_{2}\right] P, Q$ Matching \\
& & $\mid A\langle\tilde{w}\rangle$ & Recursion \\
\hline
\end{tabular}

Table 1: Syntax

process [8] modelling some protocols for pairwise node communications. Quantitative analysis in steady-state is then derived and hence the average performance indices computed. In [10] the authors define a set of metrics on the energy consumption which are then estimated through simulation and show how some changes in the protocols can improve the efficiency. With respect to the above mentioned works, the model we propose here aims at provididing a common framework for both qualitative and quantitative analysis.

Plan of the paper. Section 2 introduces the Probabilistic E-BUM calculus and its observational semantics. In Section 3 we show how to exploit the observation semantics for measuring the energy consumption of ad-hoc networks and comparing the average energy cost of networks exhibiting the same connectivity behaviour. In Section 4 we analyse the energy consumption of two well-known automatic repeat request (ARQ)-based error control protocols: stop-and-wait (SW) and go-back-N (GBN). Finally, Section 5 concludes the paper.

\section{The Calculus}

We introduce the Probabilistic E-BUM calculus, an extension of E-BUM (a calculus for Energy-aware Broadcast, Unicast, Multicast communications in mobile ad-hoc networks) [3] that models mobile ad hoc networks as a collection of nodes, running in parallel, and using channels to broadcast messages. Our calculus supports multicast and unicast communications. Moreover, it allows us to model the possibility for a node to administrate energy consumption by choosing the optimal transmission radius to communicate with the desired recipients.

Syntax. We use letters $c$ and $d$ for channels; $m$ and $n$ for nodes; $l, k$ and $h$ for locations; $r$ for transmission radii; $x, y$ and $z$ for variables. Closed values contain nodes, locations, transmission radii and any basic value (booleans, integers, ...). Values include also variables. We use $u$ and $v$ for closed values and $w$ for (open) values. We write $\tilde{v}, \tilde{w}$ for tuples of values. We write $L o c$ for the set of all locations.

The syntax of our calculus is shown in Table 1. This is defined in a two-level structure: the lower one for processes, the upper one for networks. Networks are collections of nodes (which represent devices), running in parallel, using channels to communicate messages. As usual, $\mathbf{0}$ denotes the empty network and $M_{1} \mid M_{2}$ represents the parallel composition of two networks. Processes are sequential and live within the nodes. Process $\mathbf{0}$ denotes the inactive process. Process $c(\tilde{x}) . P$ can 
receive a tuple $\tilde{w}$ of (closed) values via channel $c$ and continue as $P\{\tilde{w} / \tilde{x}\}$, i.e., as $P$ with $\tilde{w}$ substituted for $\tilde{x}$ (where $|\tilde{x}|=|\tilde{w}|)$. Process $\bar{c}_{L, r}\langle\tilde{w}\rangle . P$ can send a tuple of (closed) values $\tilde{w}$ via channel $c$ and continue as $P$. The tag $L$ is used to maintain the set of locations of the intended recipients: $L=\infty$ represents a broadcast transmission, while a finite set of locations $L$ denotes a multicast communication (unicast if $L$ is a singleton). The tag $r$ represents the power of the transmission: we assume that the choice of the transmission power may depend on precise strategies which are implemented in the communication protocol; hence it is reasonable considering the transmission radius of a communication as an information given by the process running in the sender node. Syntactically, the tags $L$ and $r$ associated with the channel $c$ in an output action may be variables, but they must be instantiated when the output prefix is ready to fire. Process $\left[w_{1}=w_{2}\right] P, Q$ behaves as $P$ if $w_{1}=w_{2}$, and as $\mathrm{Q}$ otherwise. We write $A\langle\tilde{w}\rangle$ to denote a process defined via a (possibly recursive) definition $A(\tilde{x}) \stackrel{\text { def }}{=} P$, with $|\tilde{x}|=|\tilde{w}|$ where $\tilde{x}$ contains all channels and variables that appear free in $P$. In the process $c(\tilde{x}) . P$, the variables in $\tilde{x}$ are bound in $P$. We identify processes up to $\alpha$-conversion and we assume that there are no free variables in a network. We write $c_{l}$ for $c_{\{l\}}, \bar{c}_{L, r}\langle w\rangle$ for $\bar{c}_{L, r}\langle w\rangle . \mathbf{0}$, and $\left[w_{1}=w_{2}\right] P$ for $\left[w_{1}=w_{2}\right] P, \mathbf{0}$.

Nodes cannot be created or destroyed. We write $n[P]_{l}$ for a node named $n$ located at the physical location $l$, and executing a process $P$. Each node $n$ is associated with a pair $\left\langle r_{n}, \mathbf{J}^{n}\right\rangle$, where $r_{n}$ is a non negative real number denoting the maximum transmission radius that $n$ can use to transmit, while $\mathbf{J}^{n}$ is the transition matrix of a discrete time Markov chain, where $\mathbf{J}_{l k}^{n}$ is the probabilty that the node $n$ located at $l$, after executing a movement action, will be located at $k$. Hence, $\sum_{k \in L o c} \mathbf{J}_{l k}^{n}=1$ for all locations $l \in$ Loc. Static nodes are associated with the identity Markov chain, i.e., the identity matrix $\mathbf{J}_{l l}^{n}=1$ for all $l \in L o c$ and $\mathbf{J}_{l k}^{n}=0$ for all $l \neq k$. Therefore, if the initial location of a static node is $l$, then $l$ is the only location reachable according to the node's Markov chain. In the following, we denote by $\mathcal{N}$ the set of all networks.

Nodes connectivity is verified by looking at the physical location and the transmission radius of the sender: if a node broadcasts a message, this information will be received only by the nodes that lie in the area delimited by the transmission radius of the sender. In the definition of the operational semantics we then assume the possibility of comparing locations so to determine wether a node lies or not within the transmission cell of another node. We do so by means of a function $d(\cdot, \cdot)$ which takes two locations and returns the distance separating them (the function $d$ can be simply the euclidian distance between two locations, or a more complex function considering the possible obstacles of the surrounding environment).

Probability distributions. A network $M$ is defined as the parallel composition of pairwise-distinct nodes moving independently from each other. We denote by $\prod_{i \in I} M_{i}$ the parallel composition of the networks $M_{i}$, for $i \in I$. In our framework, the mobility of the nodes is the only source of probability. We associate probability distributions with located nodes and model the probabilistic evolution of the network according to these distributions. More formally, we denote 


\begin{tabular}{ll}
\hline$n[[v=v] P, Q]_{l} \equiv n[P]_{l}$ & (Struct Then) \\
$n\left[\left[v_{1}=v_{2}\right] P, Q\right]_{l} \equiv n[Q]_{l} \quad v_{1} \neq v_{2}$ & (Struct Else) \\
$n[A\langle\tilde{v}\rangle]_{l} \equiv n[P\{\tilde{v} / \tilde{x}\}]_{l} \quad$ if $A(\tilde{x}) \stackrel{\text { def }}{=} P \wedge|\tilde{x}|=|\tilde{v}|$ & (Struct Rec) \\
$M|N \equiv N| M$ & (Struct Par Comm) \\
$(M \mid N)\left|M^{\prime} \equiv M\right|\left(N \mid M^{\prime}\right)$ & (Struct Par Assoc) \\
$M \mid \mathbf{0} \equiv M$ & (Struct Zero Par) \\
\hline
\end{tabular}

Table 2: Structural Congruence

by $\mu_{l}^{n}$ the probability distribution associated with the node $n$ located at $l$, that is a function over the set $L o c$ of locations such that, for all $k \in L o c, \mu_{l}^{n}(k)=\mathbf{J}_{l k}^{n}$ denoting the probability that the node $n$ located at $l$ moves at the location $k$.

Let $M=\prod_{i \in I} n_{i}\left[P_{i}\right]_{l_{i}}$ be a network, then for all $k$ in $I, \llbracket M \rrbracket_{\mu_{l_{k}}}^{n_{k}}$ denotes the probability distribution over the set of networks induced by $\mu_{l_{k}}^{n_{k}}$ and defined as follows: for all network $M^{\prime}$ :

$$
\llbracket M \rrbracket_{\mu_{l_{k}}^{n_{k}}}\left(M^{\prime}\right)= \begin{cases}\mu_{l_{k}}^{n_{k}}\left(l_{k}^{\prime}\right) & \text { if } M^{\prime}=\prod_{i \in I} n_{i}\left[P_{i}\right]_{l_{i}^{\prime}} \text { with } l_{i}^{\prime}=l_{i} \forall i \neq k \\ 0 & \text { otherwise }\end{cases}
$$

Note that $\llbracket M \rrbracket_{\mu_{l_{k}}}^{n_{k}}\left(M^{\prime}\right)$ is the probability that the network $M$ evolves to $M^{\prime}$ due to the movement of the node $n_{k}$ located at $l_{k}$. We say that $M^{\prime}$ is in the support of $\llbracket M \rrbracket_{\mu_{l_{k}} n_{k}}$ if $\llbracket M \rrbracket_{\mu_{l_{k}}}^{n_{k}}\left(M^{\prime}\right) \neq 0$. We write $\llbracket M \rrbracket_{\Delta}$ for the Dirac distribution on the network $M$, namely the probability distribution defined as: $\llbracket M \rrbracket \Delta(M)=1$ and $\llbracket M \rrbracket_{\Delta}\left(M^{\prime}\right)=0$ for all $M^{\prime}$ such that $M^{\prime} \neq M$. Finally, we let $\theta, \theta^{\prime}$ range over

$$
\left\{\mu_{l}^{n} \mid n \text { is a node and } l \in L o c\right\} \cup\{\Delta\} .
$$

Reduction Semantics. The dynamics of the calculus is specified by the probabilistic reduction relation over networks $(\rightarrow)$, described in Table 3. As usual in process calculi, it relies on an auxiliary relation, called structural congruence $(\equiv)$, which is the least contextual equivalence relation satisfying the rules defined in Table 2. The probabilistic reduction relation takes the form $M \rightarrow \llbracket M^{\prime} \rrbracket_{\theta}$, which describes a transition that leaves from network $M$ and leads to a probability distribution $\llbracket M^{\prime} \rrbracket_{\theta}$.

Rule (R-Bcast) models the transmission of a tuple of messages $\tilde{v}$ to the set of intended recipients $L$ using channel $c$ and transmission radius $r$. Indeed, nodes communicate using radio frequencies that enable only message broadcasting (monopolizing channels is not permitted). However, a node may decide to communicate with a specific node (or group of nodes), this is the reason why we decided to associate with each output action a set of transmission recipients. The cardinality of this set indicates the kind of communication that is used: if $L=\infty$ then the recipients set is the whole network and a broadcast transmission is performed, while if $L$ is a finite set (resp., a singleton) then a multicast (resp., a unicast) communication is realized. The recipients set indicates which are the nodes really interested in receiving that particular message, but we know that 
(R-Bcast)

$\overline{n\left[\bar{c}_{L, r}\langle\tilde{v}\rangle \cdot P\right]_{l}\left|\prod_{i \in I} n_{i}\left[c\left(\tilde{x}_{i}\right) \cdot P_{i}\right]_{l_{i}} \rightarrow \llbracket n[P]_{l}\right| \prod_{i \in I} n_{i}\left[P_{i}\left\{\tilde{v}_{i} / \tilde{x}_{i}\right\}\right]_{l_{i}} \rrbracket_{\Delta}}$

where $0<r \leq r_{n}, \forall i \in I . d\left(l, l_{i}\right) \leq r$ and $\left|\tilde{x}_{i}\right|=\left|\tilde{v}_{i}\right|$

$$
\begin{array}{r}
\text { (R-Move) } \frac{\text { (R-Par) } \frac{M \rightarrow \llbracket M^{\prime} \rrbracket_{\theta}}{M\left|N \rightarrow \llbracket M^{\prime}\right| N \rrbracket_{\theta}}}{n[P]_{l} \rightarrow \llbracket n[P]_{l} \rrbracket_{\mu_{l}^{n}}} \\
\text { (R-Struct) } \frac{M \rightarrow \llbracket M^{\prime} \rrbracket_{\theta} M^{\prime} \equiv N^{\prime}}{M \rightarrow \llbracket N^{\prime} \rrbracket_{\theta}} \\
\hline
\end{array}
$$

Table 3: Reduction Semantics

every message sent from a node will be potentially received by all the devices lying within the transmission cell of the sender. If two nodes want to share a secret, they must use cryptography to hide the message. A radius $r$ is also associated with the channel $c$, indicating the transmission radius required for that communication which may depend on the energy consumption strategy adopted by the surrounding protocol. In our calculus transmission is a non-blocking action: transmission proceeds even if there are no nodes listening for messages.

Rule (R-Move) deals with node mobility. A node $n$ located at $l$ and executing a moving action will reach a location with a probability described by the distribution $\mu_{l}^{n}$ that depends on the Markov chain $\mathbf{J}^{n}$ statically associated with $n$. Movements are atomic actions: while moving, a node cannot do anything else.

Since we are dealing with a probabilistic reduction semantics, which reduces networks into probability distributions, we need a way of representing the steps of each probabilistic evolution of a network. Formally, given a network $M$, we write $M \rightarrow_{\theta} N$ if $M \rightarrow \llbracket M^{\prime} \rrbracket_{\theta}$, and $N$ is in the support of $\llbracket M^{\prime} \rrbracket_{\theta}$. Following [?], an execution for $M$ is a (possibly infinite) sequence of steps $M \rightarrow_{\theta_{1}} M_{1} \rightarrow_{\theta_{2}} M_{2} \ldots$

In the rest of the paper, we write $\operatorname{Exec}_{M}$ for the set of all possible executions starting from $M$, last(e) for the final state of a finite execution $e$, $e^{j}$ for the prifix execution $M \rightarrow_{\theta_{1}} M_{1} \ldots \rightarrow_{\theta_{j}} M_{j}$ of lenght $j$ of the execution $e=M \rightarrow_{\theta_{1}} M_{1} \cdots \rightarrow_{\theta_{j}} M_{j} \rightarrow{ }_{\theta_{j+1}} M_{j+1} \cdots$, and $e \uparrow$ for the set of $e^{\prime}$ such that $e \leq_{\text {prefix }} e^{\prime}$. Given a network $M$ and a probability distribution $\llbracket M^{\prime} \rrbracket_{\theta}$, we write $M \rightarrow{ }^{*} M^{\prime}$ if there exists a finite execution $e \in \operatorname{Exec}_{M}$ such that last $(e)=M^{\prime}$.

Observational Semantics. The central actions of our calculus are transmission and reception of messages. However, only the transmission of messages can be observed. An observer cannot be sure whether a recipient actually receives a given value. Instead, if a node receives a message, then surely someone must have sent it. Following [7], we use the term barb as a synonymous of observable. However our calculus presents both non-deterministic and probabilistic aspects, where the non-deterministic choices are among the possible probability distri- 
butions that a process may follow and arise from the possibility for nodes to perform arbitrary, unpredicatble, movements.

We denote by behave $(M)=\left\{\llbracket M^{\prime} \rrbracket_{\theta} \mid M \rightarrow \llbracket M^{\prime} \rrbracket_{\theta}\right\}$ the set of the possible behaviours of $M$. In order to solve the non-determinism in a network execution, we consider each possible probabilistic transition $M \rightarrow \llbracket M^{\prime} \rrbracket_{\theta}$ as arising from a scheduler (see [9]). A scheduler is a total function $F$ assigning to a finite execution $e$ a distribution $\llbracket N \rrbracket_{\theta} \in$ behave (last (e)). Given a network $M$ and a scheduler $F$, we define the set of executions starting from $M$ and driven by $F$ as:

$$
\begin{gathered}
\operatorname{Exec}_{M}^{F}=\left\{e=M \rightarrow_{\theta_{1}} M_{1} \rightarrow_{\theta_{2}} M_{2} \ldots \mid \forall j, M_{j-1} \rightarrow \llbracket M_{j}^{\prime} \rrbracket_{\theta_{j}}, \llbracket M_{j}^{\prime} \rrbracket_{\theta_{j}}=F\left(e^{j-1}\right)\right. \\
\text { and } \left.\llbracket M_{j}^{\prime} \rrbracket_{\theta_{j}}\left(M_{j}\right)>0\right\} .
\end{gathered}
$$

Formally, given a finite execution $e=M \rightarrow_{\theta_{1}} M_{1} \ldots \rightarrow_{\theta_{k}} M_{k}$ starting from a network $M$ and driven by a scheduler $F$ we define

$$
P_{M}^{F}(e)=\llbracket M_{1}^{\prime} \rrbracket_{\theta_{1}}\left(M_{1}\right) \cdot \ldots \cdot \llbracket M_{k}^{\prime} \rrbracket_{\theta_{k}}\left(M_{k}\right)
$$

where $\forall j \leq k, \llbracket M_{j}^{\prime} \rrbracket_{\theta_{j}}=F\left(e^{j-1}\right)$. We define the probability space on the executions starting from a given network $M$ as follows. Given a scheduler $F, \sigma$ Field $_{M}^{F}$ is the smallest sigma field on $\operatorname{Exec}_{M}^{F}$ that contains the basic cylinders $e \uparrow$, where $e \in \operatorname{Exec}_{M}^{F}$. The probability measure $\operatorname{Prob}_{M}^{F}$ is the unique measure on $\sigma$ Field $_{M}^{F}$ such that $\operatorname{Prob}_{M}^{F}(e \uparrow)=P_{M}^{F}(e)$. Given a measurable set of networks $H$, we denote by $\operatorname{Exec}_{M}^{F}(H)$ the set of executions starting from $M$ and crossing a state in $H$. Formally $\operatorname{Exex}_{M}^{F}(H)=\left\{e \in \operatorname{Exec}_{M}^{F} \mid \operatorname{last}\left(e^{j}\right) \in H\right.$ for some $\left.j\right\}$. We denote the probability for a network $M$ to evolve into a network in $H$ according to the policy given by $F$ as $\operatorname{Prob}_{M}^{F}(H)=\operatorname{Prob}_{M}^{F}\left(\operatorname{Exec}_{M}^{F}(H)\right)$.

The notion of barb introduced below denotes an observable transmission with a certain probability according to a fixed scheduler. In our definition, a transmission is observable only if at least one location in the set of the intended recipients is able to receive the message.

Definition 1 (Barb). We say that a network $M$ has a barb on a channel c for a given scheduler $F$, written $M \downarrow_{c}^{F}$, if

i) $M \equiv n\left[\bar{c}_{L, r}\langle\tilde{v}\rangle . P\right]_{l} \mid N$

ii) there exists $k \in L$ such that $d(l, k) \leq r$

iii) $\forall e \in \operatorname{Exec}_{M}^{F}$ such that last $(e)=M F(e)=\llbracket n[P]_{l} \mid N \rrbracket_{\Delta}$.

Definition 2 (Probabilistic Barb). We say that a network $M$ has a probabilistic barb with probability $p$ on a channel $c$ according to the scheduler $F$, written $M \Downarrow_{p}^{F} c$, if $\operatorname{Prob}_{M}^{F}(H)=p$ where $H=\left\{M^{\prime} \mid M \rightarrow^{*} M^{\prime}, M^{\prime} \downarrow_{c}^{F}\right\}$.

Intuitively, for a given network $M$ and scheduler $F$, if $M \Downarrow_{p}^{F} c$ then there is a positive probability that $M$, driven by $F$, performs a transmission on channel $c$ and at least one of the intended recipients is able to correctly listen to it.

Hereafter, we introduce a probabilistic observational congruence, in the style of [?], which is parametric with respect to a set of schedulers $\mathcal{F}$ and is defined as the largest $\mathcal{F}$-relation as follows. Let $\mathcal{R}^{\mathcal{F}}$ be a relation over networks: 
Barb preservation. $\mathcal{R}^{\mathcal{F}}$ is barb preserving if $M \mathcal{R}^{\mathcal{F}} N$ and $M \Downarrow_{p}^{F} c$ for some $F \in \mathcal{F}$ implies that there exists $F^{\prime} \in \mathcal{F}$ such that $N \Downarrow_{p}^{F^{\prime}} c$.

Reduction closure. $\mathcal{R}^{\mathcal{F}}$ is reduction closed if $M \mathcal{R}^{\mathcal{F}} N$ implies that for all $F \in \mathcal{F}$, there exists $F^{\prime} \in \mathcal{F}$ such that for all classes $\mathcal{C} \in \mathcal{N} / \mathcal{R}^{\mathcal{F}}, \operatorname{Prob}_{M}^{F}(\mathcal{C})=\operatorname{Prob}_{N}^{F^{\prime}}(\mathcal{C})$.

Contextuality. $\mathcal{R}^{\mathcal{F}}$ is contextual if $M \mathcal{R}^{\mathcal{F}} N$ implies that for every context $\mathcal{C}[\cdot]$, it holds that $\mathcal{C}[M] \mathcal{R}^{\mathcal{F}} \mathcal{C}[N]$, where a context is a network term with a hole [.] defined by the grammar: $\mathcal{C}[\cdot]::=[\cdot]|[\cdot]| M|M|[\cdot]$.

Definition 3 (Probabilistic observational congruence w.r.t. $\mathcal{F}$ ). Probabilistic observational congruence w.r.t. a set $\mathcal{F}$ of schedulers, written $\cong_{p}^{\mathcal{F}}$, is the largest symmetric $\mathcal{F}$-relation over networks which is reduction closed, barb preserving and contextual.

Two networks are related by $\cong_{p}^{\mathcal{F}}$ if they exhibit the same probabilistic behaviour (communications) relative to the corresponding sets of intended recipients and the fixed set of schedulers $\mathcal{F}$. In [2] we have developed a bisimulationbased proof technique for $\cong_{p}^{\mathcal{F}}$. It provides an efficient method to check whether two networks are related by $\cong_{p}^{\mathcal{F}}$.

\section{Measuring Energy Consumption}

In this section we define a preorder over networks which allows us to compare the average energy cost of different networks but exhibiting the same connectivity behaviour. For this purpose we associate an energy cost with the probabilistic reductions as follows:

$$
\operatorname{Cost}(M, N)= \begin{cases}r \quad & \text { if } M \rightarrow \llbracket N \rrbracket_{\Delta}, M \equiv n\left[\bar{c}_{L, r}\langle\tilde{v}\rangle . P\right]_{l} \mid M^{\prime} \\ & \text { and } N \equiv n[P]_{l} \mid N^{\prime} \text { for some } c, L, v, l \\ 0 & \text { otherwise. }\end{cases}
$$

In other words, the energy cost to reach $N$ from $M$ in one single step is $r$ if $M$ can reach $N$ after firing on a channel of radius ${ }^{1} r$ regardless of the message being transmitted is observable or not (or even lost). In the same way, if $e=$ $M_{0} \rightarrow \theta_{1} M_{1} \rightarrow_{\theta_{2}} M_{2} \cdots \rightarrow_{\theta_{k}} M_{k}$ is an execution then

$$
\operatorname{Cost}(e)=\sum_{1=i}^{k} \operatorname{Cost}\left(M_{i-1}, M_{i}\right) .
$$

Let $H$ be a set of networks, we denote by $\operatorname{Path}_{M}^{F}(H)$ the set of all executions from $M$ ending in $H$ and driven by $F$ which are not prefix of any other execution ending in $H$. More formally, Paths $F_{M}^{F}(H)=\left\{e \in \operatorname{Exec}_{M}^{F}(H) \mid \operatorname{last}(e) \in\right.$ $H$ and $\forall e^{\prime}$ such that $e<_{\text {prefix }} e^{\prime}, e^{\prime} \notin$ Paths $\left._{M}^{F}(H)\right\}$.

Now, we are ready to define the average energy cost of reaching a set of networks $H$ from the initial network $M$ according to the scheduler $F$.

\footnotetext{
${ }^{1}$ Note that considering the radius of the communication channel as the energy cost of the transmitted data is standard.
} 
Definition 4. Let $H$ be a set of networks. The average energy cost of reaching $H$ from $M$ according to the scheduler $F$ is

$$
\operatorname{Cost}_{M}^{F}(H)=\frac{\sum_{e \in \operatorname{Paths}_{M}^{F}(H)} \operatorname{Cost}(e) \times P_{M}^{F}(e)}{\sum_{e \in \text { Paths }_{M}^{F}(H)} P_{M}^{F}(e)} .
$$

The average cost is computed by weighting the cost of each execution by its probability according to $F$ and normalized by the overall probability of reaching $H$.

Definition 5. Let $\mathcal{H}$ be a countable set of sets of networks and $\mathcal{F}$ be a set of schedulers. We say that $N$ is more energy efficient than $M$ w.r.t. $\mathcal{F}$ and $\mathcal{H}$,

$$
N \sqsubseteq\langle\mathcal{F}, \mathcal{H}\rangle M,
$$

if $N \cong_{p}^{\mathcal{F}} M$ and, for all schedulers $F \in \mathcal{F}$ and for all $H \in \mathcal{H}$, there exists a scheduler $F^{\prime} \in \mathcal{F}$ such that $\operatorname{Cost}_{N}^{F^{\prime}}(H) \leq \operatorname{Cost}_{M}^{F}(H)$.

\section{Analysing the SW-ARQ and GBN-ARQ Protocols}

High speed data transmission is rapidly becoming an essential requirement of today's wireless networks. Consequently, adaptive modulation and coding (AMC) techniques are increasingly being used in most of $2.5 / 3 \mathrm{~g}$ wireless networks in order to increase the transmission rate. At the same time, a wireless channel is error prone due to fading and other propagation impairments. To address this issue, many control schemes have been proposed. In particular the automatic repeat request (ARQ)-based error control is considered as very attractive to counteract the residual errors without using costly error correction codes at the physical layer (see, e.g., $[12,5])$. However, portable communication devices must rely on batteries with limited energy to conduct communication. There are three main ARQ protocols: stop-and-wait ( $S W)$, go-back-N $(G B N)$ and selective repeat (SR). In this section, we use our framework to analyse both SW-ARQ and GBN-ARQ protocols. First, we show that the protocols exhibit the same observational behaviour, that is they are bisimilar. Then, we compute and compare their energy consumption under various scenarios depending on the stability of the wireless channel.

With respect to $\mathrm{SW}$ protocols, GBN takes advantage of the pipelining of the packets, i.e., a sequence of $N$ packets can be sent without receiving any confirmation. This widely used technique is known to highly improve the throughput of the sender, but it is expensive from the energy consumption point of view [5] since correctly received packets may be required to be resent.

Modelling the Protocols. We consider a single transmitter node using ARQ-based error recovery to communicate with a receiver node over a wireless channel. Transmissions occur in fixed-size time slots. Moreover, we restrict to a one time 


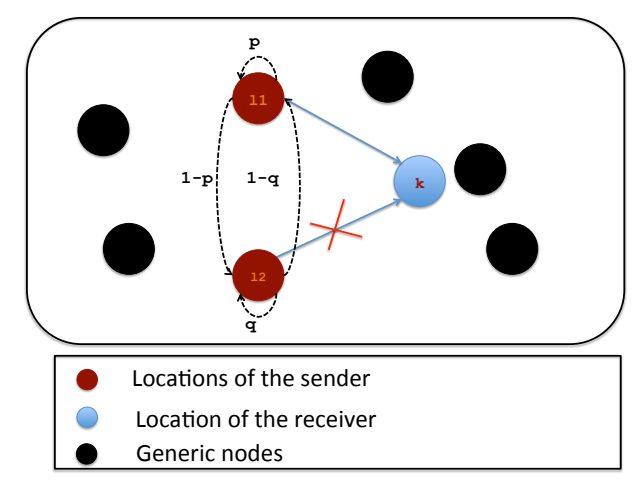

Fig. 1: Topology of the network and mobility of $s$

slot. The SW-ARQ-based protocol transmits one packet per slot while the GBNARQ-based one transmits $n$ packets (i.e., the full capacity of the channel). For both protocols, the transmitter continuously sends packets until it detects a transmission error through a NACK feedback. Here, we consider an error-free feedback channel ${ }^{2}$ and assume that the acknowledgment (ACK) or negative acknowledgment (NACK) of each transmitted packet arrives at the sender node one slot after the beginning of its transmission slot. Therefore, the feedback of a packet is received exactly after its transmission for the SW-protocol and in case of a failure (NACK), the packet is automatically resent. Instead for the GBNprotocol, a feedback for the $i$ th packet arrives exactly after the transmission of the $(i+n-1)$ th packet and in case of a failure the transmission restarts from the $i$ th packet. We model both SW-ARQ and GBN-ARQ-based protocols for a communication channel of capacity $n=3$ in our framework. We consider a unique static receiver. In order to take into account the two states nature of the channel, we model the transmitter as a mobile node send $\left(\left\langle r, J^{s}\right\rangle\right)$ whose reachable locations are $l_{1}$, which represents the "good state" of the channel, where the receiver lies within the transmission radius of the channel and $l_{2}$ the "bad state", where the destination is no longer reachable (see figure 1). The mobility of the sender is modelled by the two state Markov chain with the following transition probability matrix:

$$
J^{s}=\left|\begin{array}{cc}
p & 1-p \\
1-q & q
\end{array}\right|
$$

where $p$ and $q$ are the probabilities of the stability of the node in its good and bad states respectively.

In our analysis, we assume that the energy consumption of the feedback messages is negligible. Therefore, they are sent over channels with zero radius. For this reason the static receiver rec is located at $l_{1}$, i.e., at the same location

\footnotetext{
${ }^{2}$ A very standard assumption [5].
} 
of the sender in its good state, so that the feedback will be received with no cost. Note that the sender still transmits over channels with radius $r$ and thus consuming $r$ energy for each fired packet.

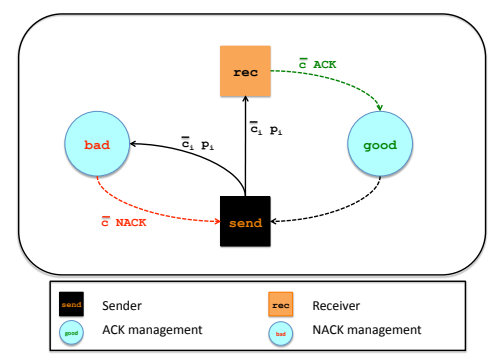

(a) Structure of the communications

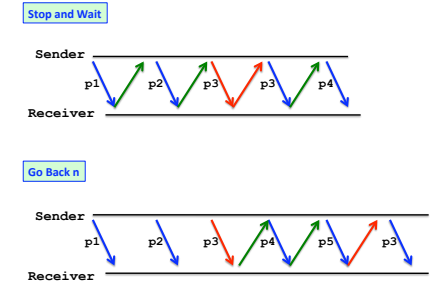

(b) Example of GBN and SW behaviour

Fig. 2: Description and example of the network communications

The process executed by $r e c$, the receiver node, is the same for both protocols and modelled as the process

$$
R E C\langle i\rangle=c_{i}(x) \cdot \bar{c}_{l_{1}, 0}\langle A C K(i)\rangle \cdot R E C\langle i+1\rangle
$$

which upon receiving packet $p_{i}$ over the channel $c_{i}$, sends $A C K(i)$ over the channel $c$, then waits for the next packet on $c_{i+1}$.

For each channel $c_{i}$, we use a static auxiliary node $b_{i}(\langle 0, I\rangle)$ located at $l_{2}$, the bad state of the sender, capturing bad transmissions over $c_{i}$. It executes the following process which upon receiving packet $p_{i}$ over the channel $c_{i}$, sends $N A C K(i)$ over the channel $c$ :

$$
B A D\langle i\rangle=c_{i}(x) \cdot \bar{c}_{\emptyset, 0}\langle N A C K(i)\rangle . B A D\langle i\rangle
$$

which upon receiving packet $p_{i}$ over the channel $c_{i}$, sends $N A C K(i)$ over the channel $c$.

GBN-ARQ. Now we introduce the full model of the protocol GBN-ARQ.

We start by modelling its sender node. Recall that, as a simplifying assumption, the channel capacity is 3 . It executes the following process:

$$
\begin{aligned}
G B\langle i\rangle= & \overline{c_{i \emptyset, r}}\left\langle p_{i}\right\rangle \cdot c\left(x_{1}\right) c_{i+1 \emptyset, r}^{-}\left\langle p_{i+1}\right\rangle \cdot c\left(x_{2}\right) c_{i+2 \emptyset, r}\left\langle p_{i+2}\right\rangle \cdot c\left(x_{3}\right) \\
& {\left[x_{1}=N A C K(i)\right] G B\langle i\rangle, S E N D\left\langle i+3, x_{2}, x_{3}\right\rangle }
\end{aligned}
$$

where the process $S E N D$ is defined as follows.

$$
S E N D\langle i, x, y\rangle=\bar{c}_{i \emptyset, r}\left\langle p_{i}\right\rangle . c(z)[x=N A C K(i-3)] G B\langle i-3\rangle, S E N D\langle i+1, y, z\rangle
$$


Though that the feedback of a packet is received after the transmission of its two successors, for practical reason, we read a feedback of a packet right after sending it. Indeed, since we do not want feedback to be costly, both sender and receiver must be located at the same place when the feedback is sent. However, the sender node will verify it only after having sent the following two packets.

Recall that the receiver node in our modelling above, reads each packet $p_{1}$ on its specific channel $c_{i}$. Thus, in the GBN, if the transmitter sends $p_{1}$ while being in its good state, then moves to bad and sends $p_{2}$ and finally moves back to the good state and sends $p_{3}$, then the later packet will not be read by the receiver as it is blocked on $c_{2}$. Then, the firing on $c_{3}$ is lost and this models the fact that packets sent after a bad packet is just a wasting of energy. But since the sender process $G B\langle i\rangle$ is blocked on the feedback channel $c$, we introduce a static auxiliary node loose $(\langle 0, I\rangle)$ located at $l_{1}$ and executing the process:

$$
W A S T=\bar{c}_{\emptyset, 0}\langle L O S T\rangle . W A S T
$$

The full model of GBN protocol is as follows.

$$
G B N=\operatorname{send}[G B\langle 1\rangle]_{l_{1}}\left|\operatorname{rec}[R E C\langle 1\rangle]_{l_{1}}\right| \operatorname{loose}[W A S T]_{l_{1}} \mid \prod_{i \geq 1} b_{i}[B A D\langle i\rangle]_{l_{2}} .
$$

SW-ARQ. Now on to the SW-ARQ-based protocol.

This is very simple since it always sends one packet and waits for its feedback. The sender process is defined as follows.

$$
S W\langle i\rangle=\bar{c}_{i \emptyset, r}\left\langle p_{i}\right\rangle \cdot c(x)[x=N A C K(i)] S W\langle i\rangle, S W\langle i+1\rangle .
$$

The full protocol is then modelled as the network

$$
S W=\operatorname{send}[S W\langle 1\rangle]_{l_{1}}\left|\operatorname{rec}[R E C\langle 1\rangle]_{l_{1}}\right| \prod_{i \in I} b_{i}[B A D\langle i\rangle]_{l_{2}} .
$$

Measuring the Energy Cost of the Protocols. This section presents the energy consumption of the above ARQ-based protocols. In order to compare the observational behaviours of the protocols, we assume that the communications over the feedback channel are observable for any observer node located at $l_{1}$. Thus the protocols are equivalent w.r.t. a set of schedulers $\mathcal{F}$ if for all schedulers $F$ in $\mathcal{F}$ driving one of the protocols, there exists a scheduler $F^{\prime}$ in $\mathcal{F}$ driving the other one such that both protocols correctly transmit the same packets with the same probabilities. Schedulers constitute an essential feature for modelling communication protocols as they provide freedom in modelling implementation and incomplete knowledge of the system. However, many schedulers could be in fact unrealistic. Consider for example schedulers giving priority to communication actions over movements of the nodes. Such schedulers cancel the two states nature of the communication channel since the latter remains in the same state until there is no longer available communication action. Thus, if the network started with a good channel then all the messages will be transmitted correctly 
without enduring any lost. In contrast, if it started with a bad channel, then it will be retransmitting indefinitely the first packet since the channel remains always bad. Though, that under such schedulers, both SW-ARQ and GBN-ARQ protocols behave exactly the same way in terms of our observability, they represent however unrealistic implementation scenarios. Therefore, we consider the following set of schedulers denoted $\mathcal{F}_{\text {alt }}$ which:

1. always alternates between sending packets and node's movement so that at each interaction of the transmitter with the channel, the later can be either good or bad;

2. gives priority to acknowledgment actions (ACK and NACK) to model the standard assumption of an error-free feedback channel;

3. allows interaction with the outside environment only through its observable actions so that we capture exactly the observable behaviour of the protocol.

Under these assumptions, we can prove the following result which shows that both protocols exhibit the same observable behaviour.

Proposition 1. GBN $\approx_{p}^{\mathcal{F}_{\text {alt }}} S W$.

We compare their energy efficiency in the context of the set $\mathcal{H}=\left\{H_{k} \mid k \geq 1\right\}$ where $H_{k}$ means that all the packets up to $k$ have been correctly transmitted and is defined as $H_{k}=H_{k}^{1} \cup H_{K}^{2}$ where

$$
\begin{gathered}
H_{k}^{1}=\left\{M\left|M \equiv \operatorname{send}\left[c_{k+1 \emptyset, r}^{-}\left\langle p_{k+1}\right\rangle \cdot P\right]_{l_{1}}\right| \operatorname{rec}[R E C\langle k+1\rangle]_{l_{1}}\right. \\
\left.\left|\operatorname{loose}[W A S T]_{l_{1}}\right| \prod_{i \geq 1} b_{i}[B A D\langle i\rangle]_{l_{2}}\right\}
\end{gathered}
$$

for some process $P$ and

$$
H_{k}^{2}=\left\{N\left|N \equiv \operatorname{send}[S W\langle i+1\rangle]_{l_{1}}\right| \operatorname{rec}[R E C\langle k+1\rangle]_{l_{1}} \mid \prod_{i \in I} b_{i}[B A D\langle i\rangle]_{l_{2}}\right\} .
$$

Then, we compute the energy consumption of the protocols assuming that we start by a move action at the good state so that the first message could be lost if it moves to the bad state ${ }^{3}$. The results are summarized in the following propositions and illustrated in Figure 3.

Proposition 2. If $q \neq 1$ then for all $F \in \mathcal{F}_{\text {alt }}$

$$
\operatorname{Cost}_{S W}^{F}\left(H_{k}\right)=\left(1+\frac{1-p}{1-q}\right) k r
$$

Proposition 3. If $q \neq 1$ then for all $F \in \mathcal{F}_{\text {alt }}$

$$
\begin{aligned}
\operatorname{Cost}_{G B N}^{F}\left(H_{k}\right)=k r(p & +\frac{(p-1)}{(-1+q)\left(1+p^{2}-q+q^{2}-p+2 p q\right)} \\
& \left.\cdot \frac{1-2 p^{2}+2 p^{2} q+4 q-4 q^{2}+2 q^{3}+2 p-6 p q+4 p q^{2}}{-p^{2}+p^{2}+(-p+p q)(-1+2 q)+q\left(2+-2 q+q^{2}\right)}\right)
\end{aligned}
$$

\footnotetext{
3 The analysis for the other case is similar.
} 


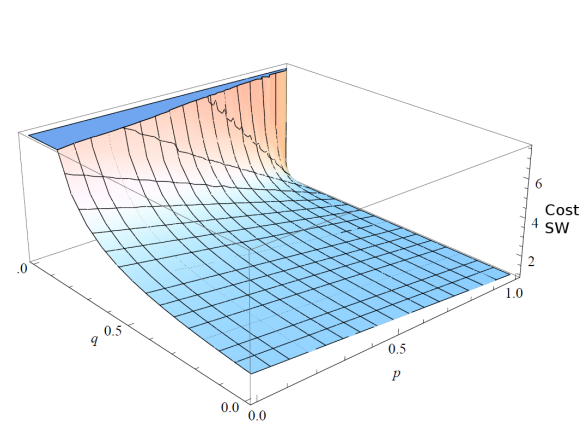

(a) SW protocol

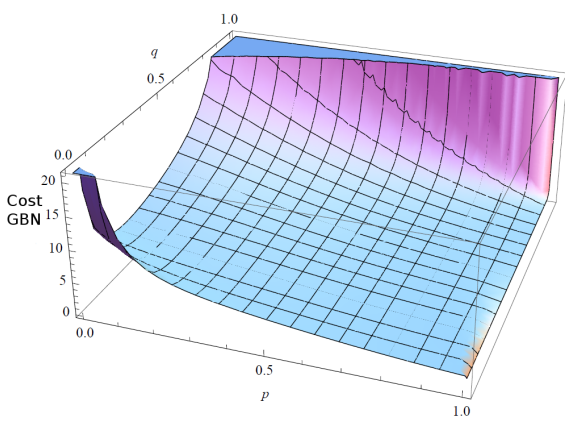

(b) GBN protocol

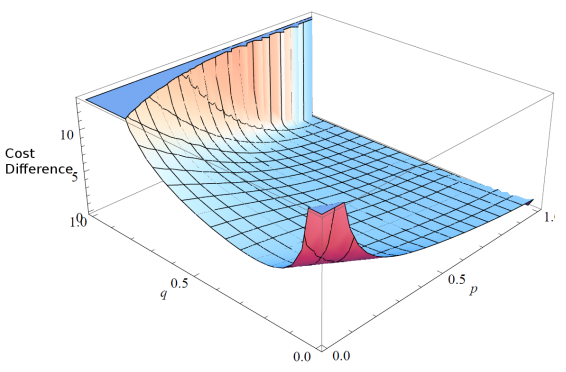

(c) $\operatorname{cost}_{G B N}(p, q)-\operatorname{cost}_{S W}(p, q)$

Fig. 3: Energy cost functions for SW and GBN protocols and their comparison.

These results can be derived by applying the Chapman-Kolmogorv's forward equations to compute the probability of consecutive failures in the sending of the same packet. Each of these failures (except the first) causes the waste of a number of sent packets equals to the window size. It can be observed that the number of wasted windows has a geometric distribution. Then, the mean of total packets sent to obtain a success, can be straightforwardly derived.

To conclude this section, we note that while both protocols increasingly enjoy bad performance in term of energy consumption when the channel deteriorates, i.e., when $q$ is increasing (see Figures 3-(a) and 3-(b)), the GBN protocol deteriorates faster. Indeed, as illustrated by Figure 3-(c) as the channel deteriorates the additional energy required by GBN protocol to correctly transmit the same number of packets increases to infinite. Thus, the gain of having a high throughput results in a very high energy consumption.

\section{Conclusion}

We presented the Probabilistic E-BUM calculus for modeling both connectivity and energy-aware properties of mobile ad-hoc networks. 
As a future work we plan to develop an observational preorder which, in one bisimulation step, checkes both observational equivalence and energy-aware preordering. We also plan to extend the model with different metrics and apply it for measuring the level of both sender- and receiver-centered interference.

\section{References}

1. M. Bernardo and M. Bravetti. Performance measure sensitive congruences for markovian process algebras. Theoretical Computer Science, 290(1):117 - 160, 2003.

2. L. Gallina, S. Hamadou, A. Marin, and S. Rossi. A probabilistic energy-aware model for mobile ad-hoc networks. Research Report DAIS-2011-3, Department of Computer Science, University Ca' Foscari of Venice, 2011.

3. L. Gallina and S. Rossi. Sender- and receiver-centered interference in wireless ad hoc networks. In Proc. of IFIP Wireless Days 2010. IEEE Computer Society Press, 2010.

4. J. Hillston. A Compositional Approach to Performance Modelling. Cambridge University Press, 1996.

5. L.B. Le, E. Hossain, and M. Zorzi. Queueing analysis for gbn and sr arq protocols under dynamic radio link adaptation with non-zero feedback delay. IEEE Transactions on Wireless Communications, 6(9):3418-3428, 2007.

6. C. Priami. Stochastic $\pi$-calculus. The Computer Journal, 38(7):578-589, 1995.

7. R.Milner and D. Sangiorgi. Barbed bisimulation. In Proc. of International Colloquium on Automata, Languages and Programming (ICALP'g2), volume 623 of LNCS, pages 685-695. Springer-Verlag, 1992.

8. S. M. Ross. Stochastic Processes. John Wiley \& Sons, 2nd edition, 1996.

9. R. Segala and N.A. Lynch. Probabilistic simulations for probabilistic processes. In Proc. of the 5th International Conference on Concurrency Theory (CONCUR '94), volume 836 of LNCS, pages 481-496. Springer-Verlag, 1994.

10. S. Singh, M. Woo, and C.S. Raghavendra. Power-aware routing in mobile ad hoc networks. In Proc. of the 4th annual ACM/IEEE International Conference on Mobile Computing and Networking (MobiCom '98), pages 181-190. ACM, 1998.

11. L. Song and J. Godskesen. Probabilistic mobility models for mobile and wireless networks. In Theoretical Computer Science, volume 323 of IFIP Advances in Information and Communication Technology, pages 86-100. Springer Boston, 2010.

12. M. Zorzi and R. R. Rao. Error control and energy consumption in communications for nomadic computing. IEEE Transactions on Computers, 46(3):279 - 289, 1997. 